Check for updates

Cite this: RSC Adv., 2017, 7, 40658

Received 17th May 2017

Accepted 15th August 2017

DOI: 10.1039/c7ra05560a

rsc.li/rsc-advances

\section{The influence of water, lanolin, urea, proline, paraffin and fatliquor on collagen $D$-spacing in leather}

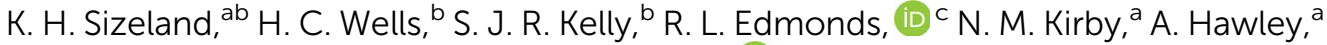 \\ S. T. Mudie, ${ }^{a}$ T. M. Ryan ${ }^{a}$ and R. G. Haverkamp (D) *b
}

\begin{abstract}
Water interacts with collagen to alter the structure at the fibrillar scale and therefore the mechanical properties of collagen. Humectants or moisturizers also alter the mechanical properties and fibril structure. The nature of these interactions and relationship between the different additives is not well understood. Changes in collagen $D$-spacing in leather were measured by synchrotron based small angle $X$-ray scattering in samples stored at various relative humidities and treated with lanolin, fatliquor, urea, proline or paraffin. The $D$-spacing increased with rising humidity and with increasing lanolin or fatliquor content, but not with treatment with urea, proline or paraffin. Strength increased with the addition of lanolin. Lanolin and fatliquor were shown to act as humectants whereas the other components did not act in this way. The Hofmeister effect is shown not to be a factor in the change in $D$-spacing, since samples treated with either proline or urea exhibited the same behavior. Different agents used in leather treatment and skin care function by different mechanisms, with collagen water retention being important for some additives but not others.
\end{abstract}

\section{Introduction}

Collagen is the main structural component of skin and other tissues, and of products derived from skin such as leather and surgical scaffolds. It imparts strength and elasticity to these materials, which are important both functionally and aesthetically. In these materials type I collagen is the main collagen present which makes up around $75 \%$ of the native tissues, and a larger portion of the processed materials.

Type I collagen has a complex hierarchical structure, and like all proteins this structure is mediated by hydrogen bonds. Type I collagen is a fibrillar protein composed of alpha helical polypeptide chains with a left-hand twist. It contains a repeating glycine- $X-Y$ amino acid sequence where a high proportion of $X$ and $Y$ positions are occupied by the amino acids proline and hydroxyproline., ${ }^{1,2}$ Three collagen molecules twist together in a right-hand manner to form a triple helix, or tropocollagen. Hydrogen bonds between side-chains stabilize the tropocollagen tertiary structure along with some covalent crosslinks. Collagen fibrils are assembled from multiples of five tropocollagens. Interchain hydrogen bonds are present as either direct or water-mediated bonds. ${ }^{3}$ The staggering of the tropocollagen in collagen fibrils produces bands, with thinner parts containing a multiple of four tropocollagens and thicker parts

${ }^{a}$ Australian Synchrotron, 800 Blackburn Road, Clayton, Melbourne, Australia ${ }^{b}$ School of Engineering and Advanced Technology, Massey University, Palmerston North, New Zealand. E-mail: r.haverkamp@massey.ac.nz

${ }^{c}$ Leather and Shoe Research Association, Palmerston North, New Zealand a multiple of five tropocollagens. This results in a d-periodicity, with characteristic $D$-spacing that is visible in transmission electron microscopy, atomic force microscopy and X-ray and neutron diffraction. ${ }^{4-6}$

Skin and other tissues are highly hydrated. Although collagen is hydrophilic and strongly associates with water, the high water content in the dermis has been attributed to the hydrophilic extracellular glycosaminoglycans also present. ${ }^{7}$ Water modifies the properties of collagen-based tissues, imparting additional suppleness and elasticity to the materials. ${ }^{8}$ Water also affects the mechanical properties of processed materials such as leather and surgical scaffolds, increasing their flexibility. ${ }^{9}$

Water has been known, from the early days of collagen structure investigation, to affect the X-ray diffraction pattern of collagen..$^{10}$ The d-periodicity of collagen decreases as collagen is dehydrated. ${ }^{11,12}$ This change in the structure of collagen may be a contributing factor in the change in its physical properties.

Suppleness may also be achieved by the application of other components. In leather "fatliquors" (usually mixtures containing polyols) are added to improve the strength and haptic properties of leather. ${ }^{13}$ Many dermatology products are used to improve the suppleness of skin. Moisturizers or emollients for dermatology may act as occlusive moisturizers (containing paraffin, petroleum jelly, cocoa butter, silicones and lanolin ${ }^{14}$ or a range of other fatty alcohols or fatty acids), blocking the evaporation of water, or as humectants (containing urea, ${ }^{15,16}$ glycerol, lactic acid, glycolic acid or salicylic acid), ${ }^{17}$ causing water retention. 
Fatliquor and lanolin have both been shown to increase the $D$-spacing of leather ${ }^{\mathbf{1 8}}$ approximately in proportion to the amount present while increasing the tear resistance. ${ }^{19}$ The addition of fatliquor increases extensibility and tear strength of leather. ${ }^{12}$

Here we investigate the mechanism by which water, in combination with moisturizing agents such as fatliquor and urea, modifies the properties of collagen by observing the changes in the collagen fibril structure.

\section{Materials and methods}

Ovine skin from the leather industry was used as a model material to investigate collagen modification by treatment with a fatliquor, Lipsol EHF (Schill + Seilacher), urea (Sigma-Aldrich), L-proline (Sigma-Aldrich), lanolin (Sigma-Aldrich) and paraffin oil (Sigma-Aldrich).

Ovine pelts were obtained from 5 month-old, early season, New Zealand Romney-cross lambs. The leather was generated using conventional beamhouse and tanning processes. Specifically, the skins were depilated using a caustic treatment comprising sodium sulfide and calcium hydroxide. The leather was then rotated in a drum for $16 \mathrm{~h}$ at $20^{\circ} \mathrm{C}$ in a $1.2 \%$ sodium sulfide solution to remove any residual keratinaceous material. The skins were next washed to remove the lime and treated with $0.1 \%$ of a commercial bate enzyme (Tanzyme, Tryptec Biochemicals, Ltd.). They were then pickled in a $2 \%$ sulfuric acid and $10 \%$ sodium chloride solution. The pickled pelts were pre-tanned using oxazolidine, degreased with an aqueous surfactant, and tanned using chromium sulfate. Finally, a 3\% vegetable tanning material (mimosa, Tanac, Brazil) was used to re-tan the resulting "wet blue".

In conventional beamhouse processing, fatliquors are added at this stage to the wet blue pelts prior to fixing with $0.5 \%$ formic acid, washing, drying and mechanical softening. The standard fatliquored samples in this study were made using $0-10 \%$ Lipsol EHF. For the other samples in this study, the fatliquor was replaced with urea or L-proline at concentrations of $4 \%$ and $7.2 \%$ by weight of wet leather, or the fatliquor was replaced with $0-10 \%$ lanolin by weight of wet leather.

The fatliquor content of samples processed with offerings of 0-10\% lipsol EHF and 0-10\% lanolin was determined using the standard method ISO 4048. $10 \mathrm{~g}$ of ground leather was extracted using at least 30 changes of dichloromethane in a Soxhlet extraction apparatus. After drying the extract in an oven at $102{ }^{\circ} \mathrm{C}$ for at least $4 \mathrm{~h}$, the resulting grease was cooled in a desiccator and weighed. The percentage fat is quoted on the basis of a moisture content in the leather at $74 \%$ relative humidity (RH).

Tear strengths of the leathers were measured using standard methods. ${ }^{20}$ Samples were cut from the leather at the official sampling position (OSP). ${ }^{21}$ The samples were then conditioned by holding at a constant temperature and humidity $\left(23^{\circ} \mathrm{C}\right.$ and $50 \%$ relative humidity) for $24 \mathrm{~h}$, after which time the samples were tested on an Instron strength-testing device.

The samples for SAXS analysis were cut from the OSP ${ }^{21}$ in strips $1 \times 30 \mathrm{~mm}$. Each sample was mounted without tension in the X-ray beam to obtain scattering patterns through the sample's full thickness. Each data point presented here is the average of 11-17 diffraction patterns recorded every $0.25 \mathrm{~mm}$ through the cross-section from the grain to the corium except the sample with no additives, which is the average of six patterns. Diffraction patterns were recorded on the Australian Synchrotron SAXS/WAXS beamline using a high-intensity undulator source. Energy resolution of $10^{-4}$ was obtained from a cryo-cooled Si (111), double-crystal monochromator, and the beam size (FWHM focused at the sample) was $250 \times 80 \mu \mathrm{m}$, with a total photon flux of about $2 \times 10^{12}$ photons $\mathrm{s}^{-1}$. All diffraction patterns were recorded with an X-ray energy of $11 \mathrm{keV}$ using a Pilatus $1 \mathrm{M}$ detector with an active area of $170 \times 170 \mathrm{~mm}$ and a sample-to-detector distance of $3371 \mathrm{~mm}$. Exposure time for diffraction patterns was $1 \mathrm{~s}$, and data processing was carried out using scatterBrain Analysis software (Australian Synchrotron).

$D$-Spacing was calculated from the position of the 6th order diffraction peak, taking the centre of a Gaussian curve fitted to the peak after subtraction of the background. Four scattering patterns were collected on each of two samples and averaged to produce one value.

Samples for SAXS analysis were maintained at controlled humidity. Untreated leather and lipsol-, lanolin-, urea-, and proline-treated leather were placed onto plastic grids in sealed containers containing one of a saturated solution of potassium acetate, sodium bromide, or sodium chloride. These yielded $22.5 \%, 57.6 \%$, and $75.3 \%$ relative humidities, respectively. The samples were allowed to equilibrate at these humidities for $48 \mathrm{~h}$ prior to SAXS analysis. Samples were also soaked in water. These are shown in the results as " $100 \%$ relative humidity" and plot them on the scale at this point, but they are saturated with water and contain free water. The samples were sealed in kapton tape prior to analysis to ensure they remained as close as possible to their humidity-equilibrated state.

\section{Results}

\section{Scattering patterns}

SAXS patterns were obtained for the samples processed with lipsol, lanolin, urea, and proline. Diffraction rings that occur due to the axial periodicity displayed by collagen were clearly visible in every spectrum (Fig. 1a). From the varying intensity around the azimuthal angle of the rings, the orientation of the fibrils can be determined. The integrated intensity of the scattering pattern can be plotted (Fig. 1b) and the $D$-spacing can be calculated using the central position of the diffraction peak divided by the peak order.

\section{Lanolin content}

The leather was treated with lanolin at concentrations of 2, 4, 6, 8 and $10 \%$. Samples had about $0.4 \%$ initial fat content and the lanolin was taken up completely with increasing concentration up to about $6 \%$ (Table 1). Over $6 \%$, the uptake of lanolin was not complete. 

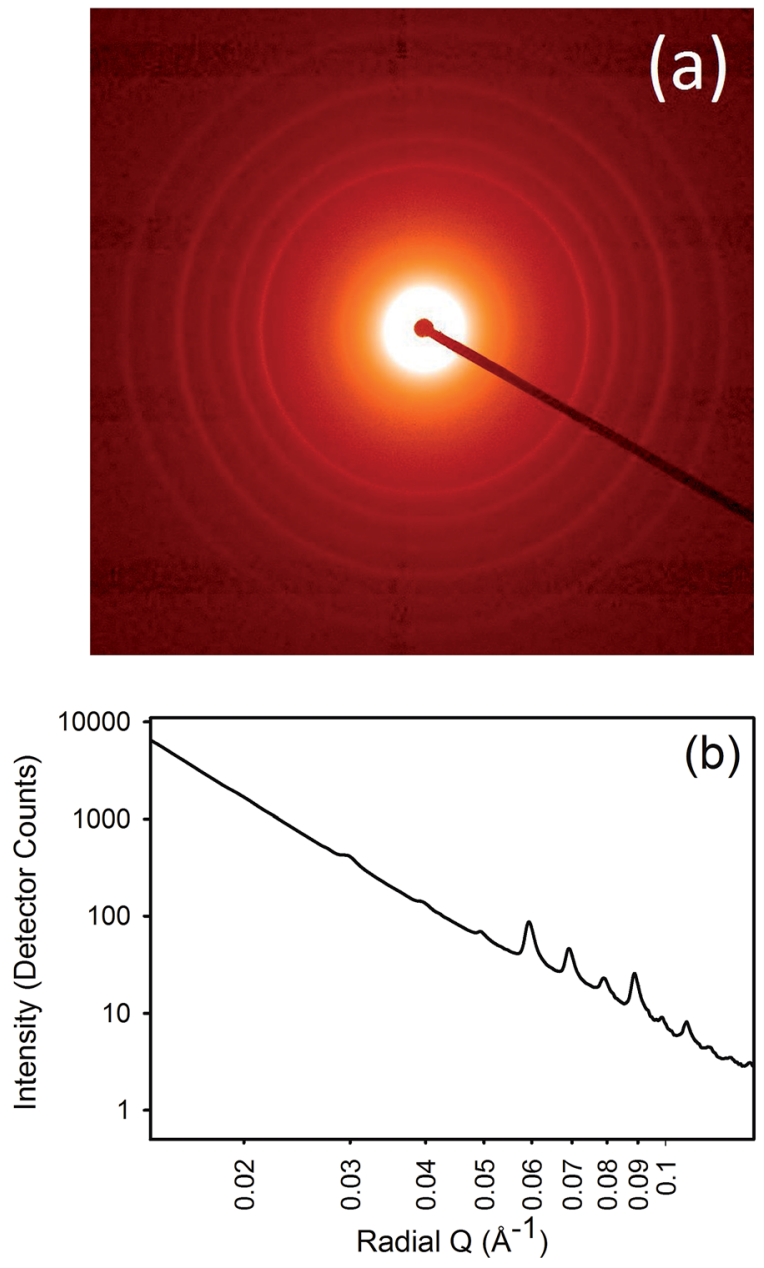

Fig. 1 Example of SAXS of collagen: (a) SAXS pattern; (b) integrated intensity profile.

Table 1 Measured fat content of leather after lanolin addition. This includes the lanolin and other fats already present

\begin{tabular}{ll}
\hline Lanolin offer & Fat in wet blue \\
\hline $0 \%$ & $0.4 \%$ \\
$2 \%$ & $2.4 \%$ \\
$4 \%$ & $4.7 \%$ \\
$6 \%$ & $6.4 \%$ \\
$8 \%$ & $7.4 \%$ \\
$10 \%$ & $7.9 \%$ \\
\hline
\end{tabular}

\section{Lipsol content}

The leather was treated with lipsol at concentrations of 2, 4, 6, 8 and $10 \%$. The lipsol was taken up completely by the leather up to concentrations of $8 \%$ (Table 2). At $10 \%$ lipsol, there was no increase in fat content above that caused by treatment with $8 \%$ lipsol so the uptake of lipsol was not complete.

\section{$D$-Spacing and relative humidity}

$D$-Spacing increased with increasing relative humidity for samples treated with either lanolin or fatliquor (Fig. 2). $D$ -
Table 2 Measured fat content of leather after Lipsol addition. This includes the Lipsol and other fats already present

\begin{tabular}{ll}
\hline Lipsol EHF offer & Fat in wet blue \\
\hline $2 \%$ & $4 \%$ \\
$4 \%$ & $6 \%$ \\
$6 \%$ & $9 \%$ \\
$8 \%$ & $10 \%$ \\
$10 \%$ & $10 \%$
\end{tabular}
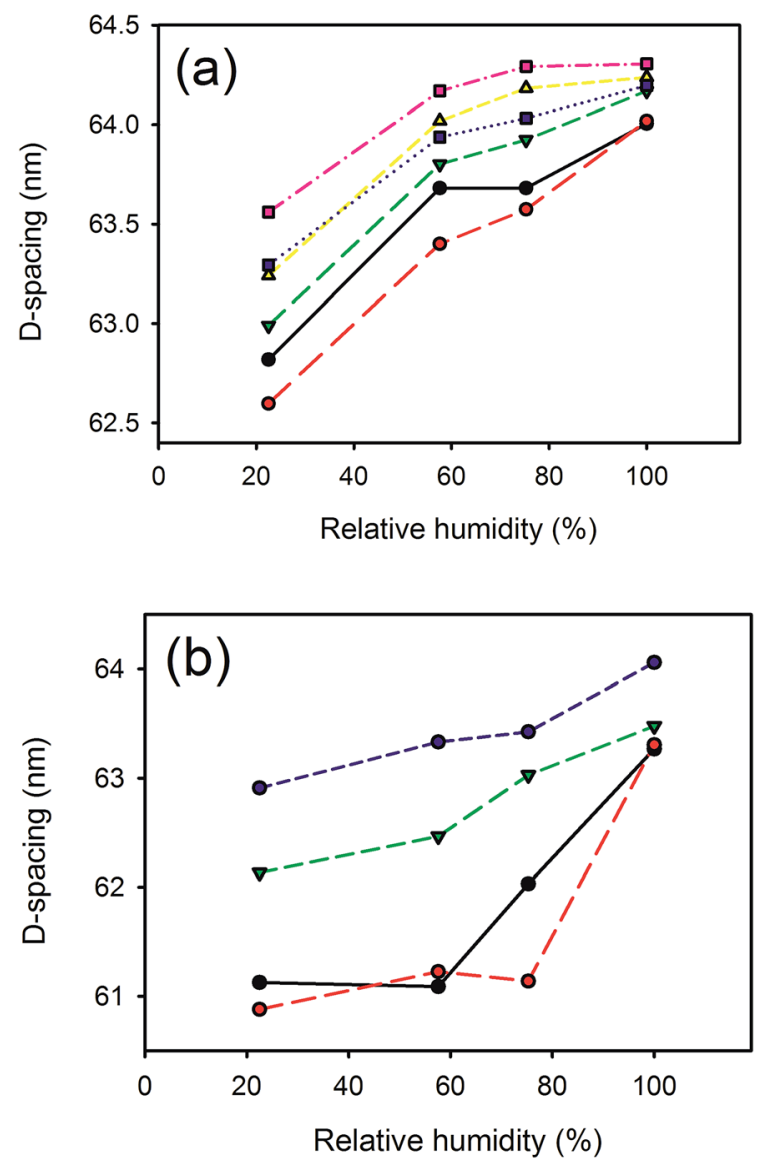

Fig. 2 Relative humidity versus $D$-spacing for: (a) lanolin $(0 \%-$, $2 \%--, 4 \%--, 6 \% \ldots, 8 \% \cdots \cdots, 10 \%+\cdots,-.-$ ) ( (b) Lipsol EHF $(0 \%-$, $2 \%---, 6 \%---, 8 \%---)$.

Spacing in the former samples increased to almost converge at $100 \%$ relative humidity. The $D$-spacing of lipsol-treated samples increased slightly from $22.5 \%$ relative humidity to $57.6 \%$ relative humidity, before increasing to almost converge at $100 \%$ humidity. It is notable that the leather containing $2 \%$ lanolin and the leather containing $2 \%$ lipsol are out of sequence and we do not have an explanation for this.

\section{$D$-Spacing and lanolin content}

$D$-Spacing increased with increasing lanolin or fatliquor content (Fig. 3). The effect was much greater at low humidity, and the $D$-spacing was rather insensitive to lanolin or fatliquor treatment at higher humidity. 

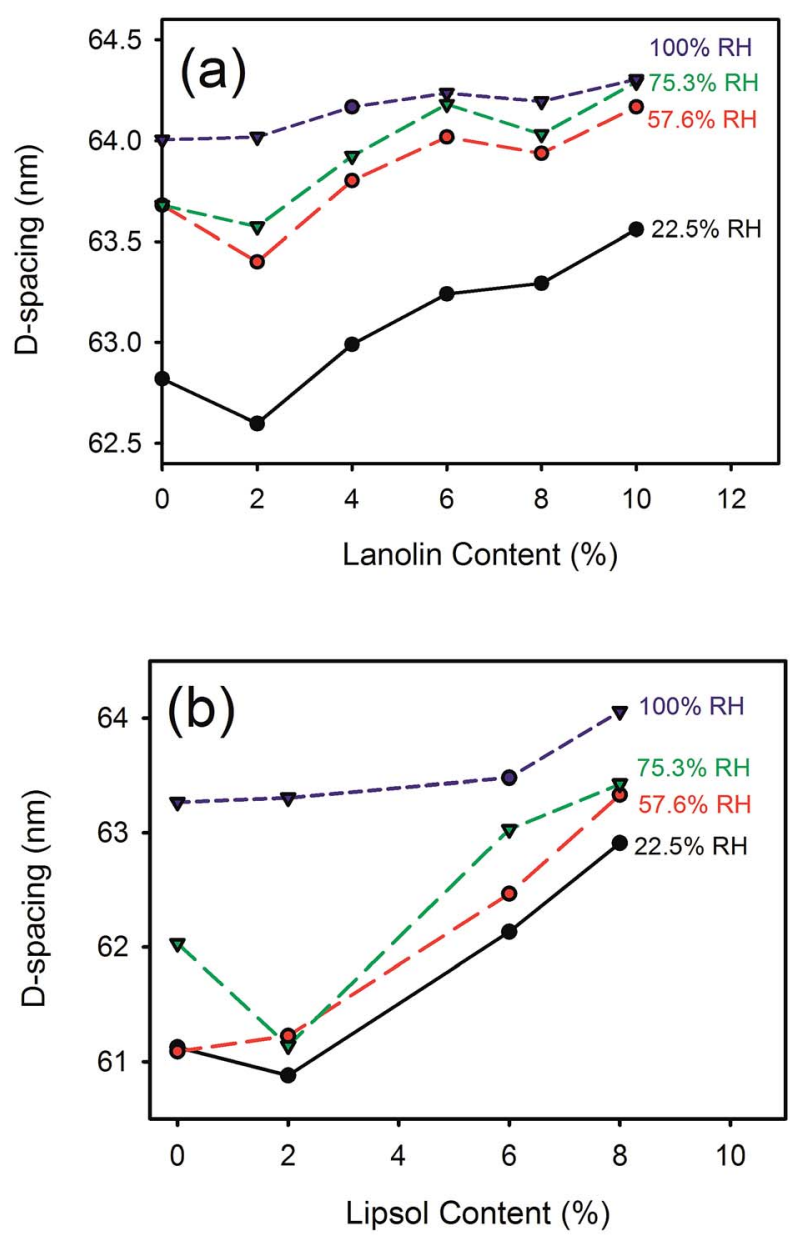

Fig. 3 D-Spacing with (a) lanolin or (b) Lipsol EHF fatliquor content at different relative humidities.

\section{$D$-Spacing and paraffin content}

The $D$-spacing for leather treated with paraffin was similar or lower than that of the control leather samples (Fig. 4).

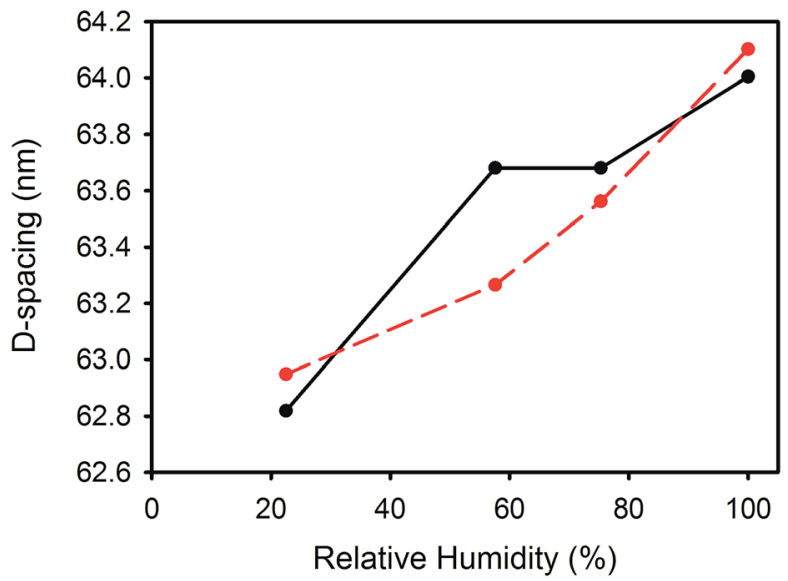

Fig. 4 Paraffin in leather $(-)$, and control $(---)$ at different relative humidities.
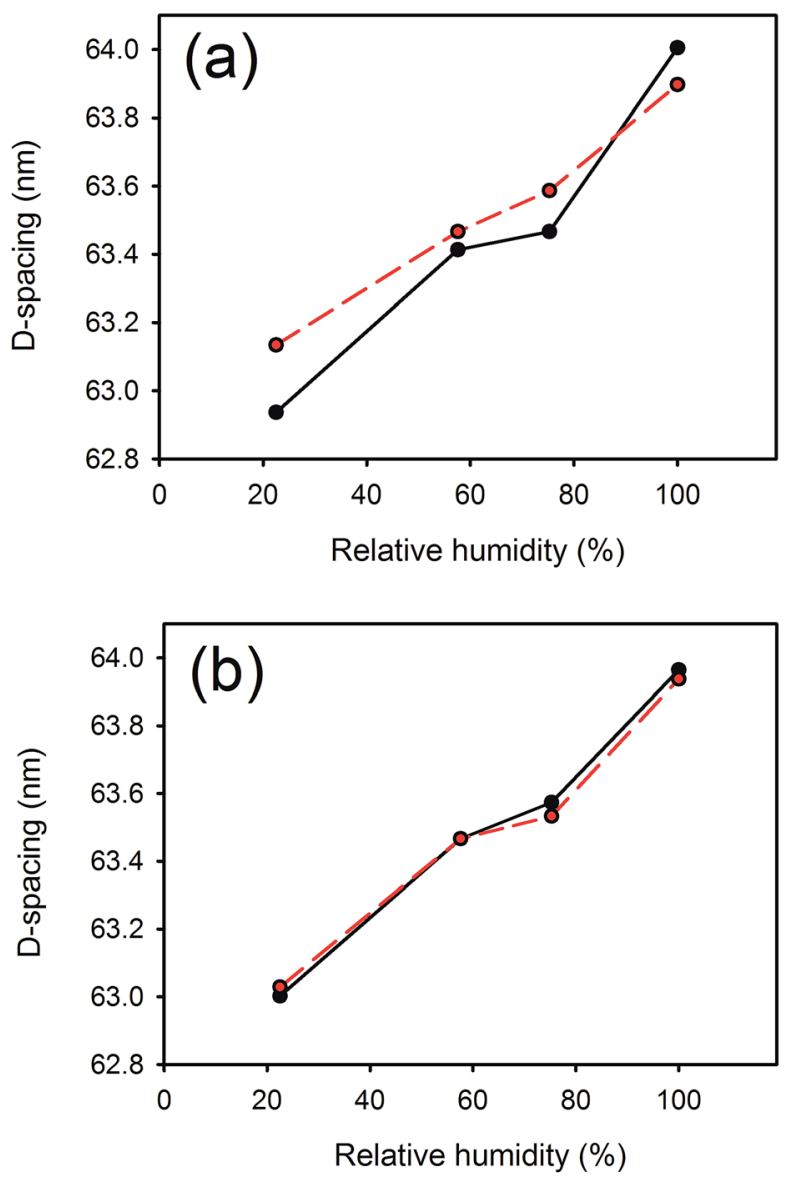

Fig. 5 Relative humidity versus $D$-spacing for: (a) urea $(4 \%-$, $7.2 \%---)$; (b) proline $(4 \%-, 7.2 \%---)$.

\section{$D$-Spacing and urea and proline content}

The $D$-spacing was higher in samples treated with $7.2 \%$ urea compared with samples treated with $4 \%$ urea at all humidities except for $100 \%$ relative humidity. For samples exposed to those two treatments, the $D$-spacing increased with increasing relative humidity. The $D$-spacing was similar between samples treated

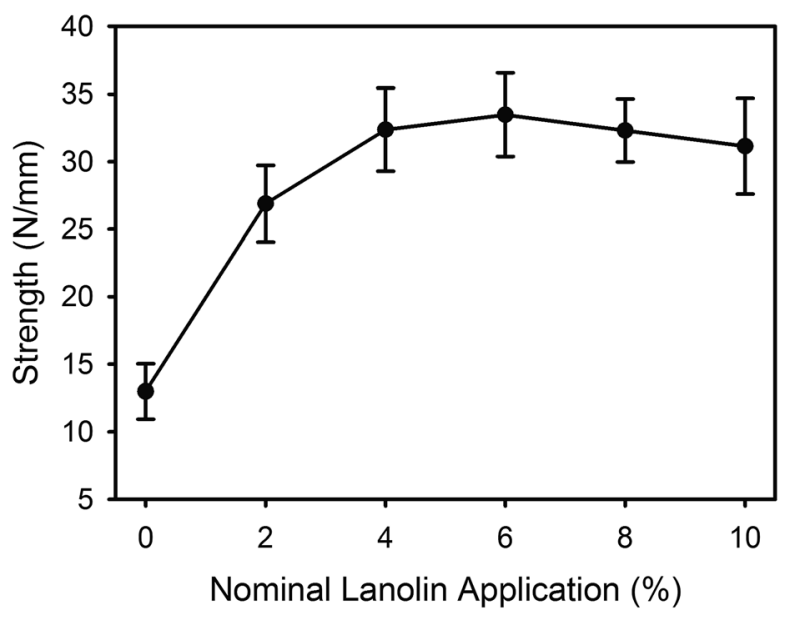

Fig. 6 Tear strength of leather with lanolin content. 
with $4 \%$ or $7.2 \%$ proline, and, similar to the urea-treated samples, the $D$-spacing increased with increasing humidity (Fig. 5).

\section{Tear strength}

The tear strength of the leather increased with the addition of lanolin, with the greatest increase occurring at the lower levels of lanolin addition (Fig. 6).

\section{Discussion}

The collagen $D$-spacing increases when the collagen is more hydrated. That there is a difference between wet and dry collagen is well known, ${ }^{\mathbf{1 0 , 1 1}}$ and here we have shown that this is not a binary phenomenon but rather that there is a steady increase in $D$-spacing with increasing humidity. The change observed here with hydration is up to $2.4 \mathrm{~nm}$ for the $D$-spacing or $4 \%$ in the length.

However, the increase in $D$-spacing with humidity is affected very substantially by other treatment to which the leather has been exposed. It has previously been shown that lanolin or fatliquor increases the $D$-spacing of collagen. ${ }^{12,19}$ When more lanolin is present in leather, the change in $D$-spacing with increasing moisture content is smaller (Fig. 2 and 3). The $D$ spacing almost converges with that of the wet material, regardless of the amount of lanolin present. A similar trend is observed for the fatliquor Lipsol. It is possible that this effect is due to a combined effect of lanolin increasing the $D$-spacing and water increasing the $D$-spacing, such that the upper limit of $D$-spacing is approached. However, another possible explanation is that the $D$-spacing increase is due only to an increase in water, and that the lanolin or fatliquor contributes to the retention of water, so that at a given humidity more water is retained when lanolin is present. Lanolin (and fatliquors) is an amphoteric compound, having affinity to both polar and nonpolar molecules, so it can facilitate water retention.

It could be argued that lanolin's oily nature contributes to increases in $D$-spacing rather than its amphoteric nature. To test this hypothesis, a comparison was made with the effect of a purely hydrophobic chemical, paraffin oil, on collagen $D$ spacing. While increases in humidity were still associated with increased $D$-spacing, the addition of paraffin oil did not enhance this effect, and at lower humidity it had the opposite effect, that of reducing the $D$-spacing (Fig. 5), perhaps due to its hydrophobic nature excluding water. This result supports the notion that it is not the lanolin directly that causes the increase in $D$-spacing, but rather the lanolin's water-retaining capacity that does so. So while lanolin is generally considered an occlusive agent in skin care, ${ }^{\mathbf{1 4 , 1 7}}$ it appears from the observed increases in $D$-spacing of collagen that lanolin is a humectant.

Urea is commonly used as an ingredient in dermatology products, ${ }^{15,16}$ and it is also known to be near one end of the Hofmeister series as a denaturant of proteins. ${ }^{22}$ Denaturing protein involves the disruption of hydrogen bonds that provide structural form to the molecular backbone. It has been hypothesized that urea could act on collagen to weaken the hydrogen bonds and therefore expand the fibril structure (with an increase in $D$-spacing), and that this might provide an increase in fibril length and therefore expanded and smoother skin. However, the measurement of $D$-spacing of samples treated with urea does not confirm this hypothesis. While the $D$ spacing increased with increasing humidity it did not appear to increase with increasing urea content (Fig. 5), although the data here are rather limited and preliminary. Proline is at the other end of the Hofmeister series, and acts to precipitate proteins from solution. ${ }^{23}$ If this effect influences $D$-spacing then proline might be thought to decrease the $D$-spacing, but this was not observed. The lack of effect on $D$-spacing of both urea and proline suggests that the Hofmeister theory is not relevant to this phenomenon. Both urea and proline are hydrophobic ${ }^{24}$ and it is this property (the lack of amphoteric or polar nature) that may be the main factor leading to a lack of influence on the collagen $D$-spacing. This leaves unanswered, therefore, the question of why urea is a useful skin cream additive.

The tear strength of leather is increased with the addition of lanolin (Fig. 6) but this may be due to the lanolin's ability to enhance water retention, as the level of hydration of leather is known to affect the tear strength. ${ }^{9}$

These changes in $D$-spacing observed in collagen as a result of water content and facilitated by various additives may be related to the physical properties of leather and skin in two or more ways. An increase in $D$-spacing means longer collagen fibrils and an increase in length of a few percent may help to remove wrinkles in skin. However, an increase in length does not necessarily translate to a stronger or more supple material. The higher level of hydration that a higher $D$-spacing reflects may be the cause of the improved mechanical properties of skin and leather. Tropocollagen molecules are linked by extensive hydrogen bonds, ${ }^{3}$ and this hydrogen bonding requires hydration. A greater level of hydration may impact on this hydrogen bonding and the ability of the tropocollagen to move relative to neighbouring tropocollagens or for fibrils to move relative to other fibrils. In skin this may also be mediated by glycosaminoglycans. ${ }^{25}$

\section{Conclusions}

It has been shown in leather that the collagen $D$-spacing increases with increasing humidity. Treatment with increasing amounts of amphoteric compounds such as lanolin or fatliquor also increases the $D$-spacing, and this may be due to the waterretaining properties of these components. Paraffin oil is hydrophobic and treatment with it does not promote water retention in leather, nor does it increase the $D$-spacing of collagen. The Hofmeister effect has been shown not to be a contributor to changes in $D$-spacing, with the hydrophobic molecules urea and proline (at opposite ends of the Hofmeister series) not changing the $D$-spacing of collagen. While it has been shown what influence a range of treatment agents has on the $D$-spacing of collagen, this has resulted in some unanswered questions about the influence of $D$-spacing on the properties of leather and skin, and the function of chemicals that are commonly used in skin care products, some of which retain water and increase the $D$-spacing of collagen and others which do not. 


\section{Conflicts of interest}

There are no conflicts of interest to declare.

\section{Acknowledgements}

This research was undertaken on the SAXS/WAXS beamline at the Australian Synchrotron in Victoria, Australia who provided beamtime and travel funding. The leathers examined in this work were prepared with funding from the New Zealand Ministry of Business and Innovation grant LSRX1301.

\section{References}

1 J. A. M. Ramshaw, N. K. Shah and B. Brodsky, J. Struct. Biol., 1998, 122, 86-91.

2 M. D. Shoulders and R. T. Raines, Annu. Rev. Biochem., 2009, 78, 929-958.

3 J. Bella, M. Eaton, B. Brodsky and H. M. Berman, Science, 1994, 266, 75-81.

4 T. J. Wess, A. Miller and J. P. Bradshaw, J. Mol. Biol., 1990, 213, 1-5.

5 P. Fratzl, K. Misof, I. Zizak, G. Rapp, H. Amenitsch and S. Bernstorff, J. Struct. Biol., 1997, 122, 119-122.

6 M. M. Basil-Jones, R. L. Edmonds, T. F. Allsop, S. M. Cooper, G. Holmes, G. E. Norris, D. J. Cookson, N. Kirby and R. G. Haverkamp, J. Agric. Food Chem., 2010, 58, 5286-5291. 7 N. J. Raine-Fenning, M. P. Brincat and Y. Muscat-Baron, Am. J. Clin. Dermatol., 2003, 4, 371-378.

8 Y. S. Papir, K. H. Hsu and R. H. Wildnauer, BBA, Biochim. Biophys. Acta, Gen. Subj., 1975, 399, 170-180.

9 M. Komanowsky, J. Am. Leather Chem. Assoc., 1990, 85, 6-18.
10 A. Rich and F. H. C. Crick, J. Mol. Biol., 1961, 3, 483-506. 11 Y. F. Huang and K. M. Meek, Biophys. J., 1999, 77, 1655-1665. 12 K. H. Sizeland, H. C. Wells, G. E. Norris, R. L. Edmonds, N. Kirby, A. Hawley, S. Mudie and R. G. Haverkamp, J. Am. Leather Chem. Assoc., 2015, 110, 66-71.

13 C. K. Liu, N. P. Latona and G. L. DiMaio, J. Am. Leather Chem. Assoc., 2002, 97, 284-293.

14 I. Harris and U. Hoppe, Dry Skin Moisturizers, 2000, 259-267. 15 M. Pan, G. Heinecke, S. Bernardo, C. Tsui and J. Levitt, Dermatol. Online J., 2013, 19, 20392.

16 M. Loden, Dry Skin and Moisturizers, 2000, 243-250.

17 T. C. Flynn, J. Petros, R. E. Clark and G. E. Viehman, Clin. Dermatol., 2001, 19, 387-392.

18 K. H. Sizeland, H. C. Wells, G. E. Norris, R. L. Edmonds, N. Kirby, A. Hawley, S. Mudie and R. G. Haverkamp, J. Am. Leather Chem. Assoc., 2015, 110, 66-71.

19 K. H. Sizeland, G. Holmes, R. L. Edmonds, N. Kirby, A. Hawley, S. T. Mudie and R. G. Haverkamp, J. Am. Leather Chem. Assoc., 2015, 110, 355-362.

20 J. M. V. Williams, J. Soc. Leather Technol. Chem., 2000, 84, 327-329.

21 J. M. V. Williams, J. Soc. Leather Technol. Chem., 2000, 84, 303-309.

22 W. Kunz, Curr. Opin. Colloid Interface Sci., 2010, 15, 34-39.

23 M. T. Record, E. Guinn, L. Pegram and M. Capp, Faraday Discuss., 2013, 160, 9-44.

24 R. Wolfenden, C. A. Lewis Jr, Y. Yuan and C. W. Carter Jr, Proc. Natl. Acad. Sci. U. S. A., 2015, 112, 7484-7488.

25 H. R. Kayed, K. H. Sizeland, N. Kirby, A. Hawley, S. T. Mudie and R. G. Haverkamp, RSC Adv., 2015, 5, 103703-103712. 